\title{
Les ambivalences de la mise en tourisme du patrimoine. Le cas du centre ancien de Tozeur (Tunisie)
}

Najem Dhaher

\section{OpenEdition}

Journals

Édition électronique

URL : http://journals.openedition.org/tourisme/232

DOI : 10.4000/tourisme.232

ISSN : 2492-7503

Éditeur

Éditions touristiques européennes

\section{Édition imprimée}

Date de publication : 1 décembre 2012

Pagination : 23-33

ISSN : 2109-5671

\section{Référence électronique}

Najem Dhaher, «Les ambivalences de la mise en tourisme du patrimoine. Le cas du centre ancien de Tozeur (Tunisie) », Mondes du Tourisme [En ligne], 6 | 2012, mis en ligne le 30 septembre 2015 consulté le 04 mai 2019. URL : http://journals.openedition.org/tourisme/232 ; DOI : 10.4000/ tourisme.232

\section{(c) (1) $\odot$}

Mondes du tourisme est mis à disposition selon les termes de la licence Creative Commons Attribution - Pas d'Utilisation Commerciale - Pas de Modification 4.0 International. 


\section{Les ambivalences}

\section{de la mise en tourisme du patrimoine.}

\section{Le cas du centre ancien de Tozeur (Tunisie)}

NAJEM DHAHER

[najem_dhaher@yahoo.fr]

Docteur en urbanisme

Maitre assistant à l'École nationale d'architecture et d'urbanisme de Tunis, Université de Carthage

Résumé. Longtemps ignorée, la question de la réhabilitation et de la sauvegarde du patrimoine est devenue une des préoccupations des acteurs aussi bien publics que privés en Tunisie. L'augmentation de la fréquentation touristique des monuments et des sites historiques sahariens et l'attachement des populations à ces signes de leur identité ont contribué à l'intérêt croissant que leur portent les différents acteurs urbains. Cet intérêt, qui se traduit par des travaux de restauration et de mise en valeur des quartiers historiques, témoigne du nouveau potentiel de croissance économique que représente le patrimoine pour le développement local. Pourtant, la mise en œuvre d'une véritable politique de patrimonialisation et de réhabilitation révèle des enjeux sociétaux liés à la fois à ce processus de prise en compte du patrimoine et aux techniques qui concernent les savoir-faire.

Aujourd'hui, alors que le tourisme paraît être le seul espoir de sortie de crise pour certains territoires, le patrimoine architectural de la ville de Tozeur est devenu un enjeu économique. II constitue un pilier important pour l'industrie du tourisme. La situation actuelle est marquée par une sorte d'antagonisme entre un phénomène de prise de conscience de l'importance économique du patrimoine, qui s'est fait jour depuis quelques années, et un comportement purement mercantile qui s'est ensuivi. Aujourd'hui, les pouvoirs locaux sont-ils arrivés à concilier sauvegarde et utilisation économique du patrimoine ?

Abstract. For a long time ignored, the question of the rehabilitation and the protection of the heritage became one of the preoccupations of actors so public as private in Tunisia. The increase of the tourist attendance of monuments and of the Saharan historic sites and the attachment of the populations to these signs of their identity contributed to the increasing interest which carry them the various urban actors. This interest which is translated by works of restoration and development of the historic districts testifies of the new potential of growth that heritage represents for the local development. Nevertheless, the implementation of a real politics of patrimonialisation and of rehabilitation reveals at once societal stakes bound to the patrimonialisation and to the techniques which concern the know-how.

Today, while the tourism appears the only hope of exit of crisis for certain places, the architectural heritage in the city of Tozeur became an economic stake. It constitutes a pillar important for the industry of the tourism. The current situation is marked by a kind of antagonism enter a phenomenon of awareness of the economic importance of the heritage which was been daylight for some years and a purely mercantile behavior which followed itself. Today, the local powers managed to reconcile saving and economic use of the heritage? 
$\mathbf{A}$ ujourd'hui, le patrimoine est devenu un enjeu économique et s'inscrit au cœur du débat sur le développement local. Il constitue un élément important tant pour l'industrie du tourisme que pour celle de la culture. L'augmentation de la fréquentation des monuments et des sites, l'attachement des populations à ces signes de leur identité, l'intérêt croissant que leur portent les autorités locales - qui se traduit par des dispositifs de conservation et de mise en valeur - témoignent du potentiel que représente le patrimoine pour le développement local. L'architecture domestique des quartiers historiques, leur réalité sociale et leur place dans le fonctionnement des agglomérations contemporaines ainsi que les problèmes posés par leur sauvegarde ont souvent fait l'objet de recherches menées sur le patrimoine bâti. Dans un pays comme la Tunisie, à faibles ressources naturelles et minières mais au passé important, la mise en valeur du patrimoine architectural à des fins touristiques est considérée comme un outil majeur de développement et de promotion des territoires. Aujourd'hui, alors que le développement du tourisme prend des dimensions notables, la réflexion sur la patrimonialisation et la préservation des sites historiques est devenue d'actualité, voire nécessaire. Le regain d'intérêt pour les sites historiques est souvent orienté vers une perspective de développement touristique. En Tunisie, l'investissement public et privé dans les quartiers historiques à travers des projets tou- ristiques à valeur culturelle et patrimoniale est récent. Dans la ville saharienne de Tozeur, réputée pour sa culture, ses traditions et son patrimoine architectural très particulier, le tourisme a suscité ces dernières années une réflexion autour de la notion de patrimoine. Cela a conduit à des opérations de restauration de certaines parties de la ville, notamment les plus anciennes, en vue d'attirer les flux touristiques.

Le patrimoine culturel à Tozeur constitue une composante essentielle de la ville, et la tentation d'en faire un élément moteur du développement local est grande et justifiée. Surtout avec une activité touristique qui est en pleine expansion au cœur des processus de valorisation des territoires urbains. Mais la valorisation du patrimoine, qui s'inscrit de plus en plus dans une "démarche produit", laisse penser que cette action risque d'aboutir à sa banalisation voire à sa "folklorisation". En réalité, le tourisme, qui a besoin de culture, peut paradoxalement engendrer la destruction de cette dernière comme dans le cas des processus de patrimonialisation, voire de muséification des centres anciens dont les fonctions urbaines traditionnelles sont temporairement ou définitivement sacrifiées au profit de leur mise en tourisme. En effet, si certaines évolutions ont animé les espaces traditionnels de Tozeur, favorisant l'apparition de mécanismes de participation du patrimoine à la croissance économique et touristique des territoires, des dysfonctionnements ont également caractérisé les opérations de sauvegarde et de mise en valeur entreprises.

Par des approches variées, cette réflexion s'appuiera sur le cas typique du centre historique de la ville de Tozeur, empreint d'une forte tradition culturelle, pour dévoiler le caractère marchand du patrimoine et comprendre les mécanismes et les conséquences de la mise en tourisme. L'analyse est centrée sur les transformations architecturales en relation avec le tourisme et la patrimonialisation. Entre la conservation et la mise en tourisme, entre l'universalité de la valeur du patrimoine et sa fonctionnalité, sa pérennisation et son rôle dans la société actuelle, il s'avère nécessaire de comprendre comment on peut réussir la "touristification" du patrimoine pour qu'il joue un rôle dans le développement local.

\section{LE CENTRE DE TOZEUR \\ Un patrimoine qui se prête} à la consommation touristique

La ville de Tozeur se trouve à 450 kilomètres au sud-ouest de la capitale Tunis. Elle était une sorte de port du désert, un centre actif du commerce caravanier transsaharien. L'histoire de la structuration de son espace, à l'instar de toutes les villes du Sud tunisien, est liée à celle des différents groupes venus à un moment ou à un autre s'installer dans la ville. Les Berbères sont les premiers habitants de la ville, qui a été conquise par les Romains en l'an 33 av. J.-C., puis par les Arabes 
dès le début de la diffusion de l'islam. Appelée Thusuros, la ville représentait un point fort de contrôle sur le limes de l'Empire romain, s'ouvrant sur le grand Sahara. Avec l'arrivée des musulmans au VII siècle, l'agriculture oasienne et le commerce caravanier connaissent leur apogée (Kassah, 1996).

Après la destruction de la ville par les rebelles Hafsides (XIII siècle), une majorité de la population est revenue la reconstruire. Ainsi, les premières installations furent bâties à l'extérieur de l'oasis. Depuis le début $d u X I^{e}$ siècle, la ville a été constituée de différents noyaux qui se sont formés à des époques différentes et qui relèvent d'une certaine construction sociale et des représentations des habitants. Au XVI ${ }^{e}$ siècle, El Hadef ${ }^{(1)}$ vient habiter Tozeur et devient le véritable chef de la ville. La famille d'El Hadef s'est implantée dans un regroupement de houchs ${ }^{(2)}$ accolés suivant une structure appuyée sur des rues étroites, des impasses et des placettes babbouha. Les houchs ouvrent sur ces éléments urbains et constituaient un tissu compact. Le lieu de rassemblement des caravanes reste le même, mais le lieu d'échanges se déplace devant le quartier de Ouled El Hadef, qui, selon Paty de Clam (1890), prend de plus en plus d'importance. L'arrivée au XVII siècle des Zebda (familles d'origine arabe) a été accompagnée de la formation d'un nouveau groupement urbain, semblable à celui des Ouled El Hadef mais beaucoup plus régulier. Les Ouled Sidi Abid, arrivés aussi au XVII siècle, s'installaient au nordouest du quartier Ouled El Hadef avec qui ils devenaient des alliés.

La présence coloniale européenne, du XIX au XXe siècle, eut quelques effets déstructurants sur l'organisation spatiale traditionnelle sans qu'un nouveau modèle lui soit substitué malgré la politique de sédentarisation forcée des nomades dans la ville et notamment la construction de quelques bâtiments administratifs. Après l'indépendance du pays, en 1956, l'extension de la ville s'est faite surtout en direction du nord et de l'est par la création de zones d'habitation populaires, qui ont reconduit le modèle d'habitat bédouin spécifique, que l'on observe dans le Sud tunisien, sans schéma conducteur ni planification urbaine. La croissance démographique qui a rendu difficile le maintien dans ces quartiers d'une partie de leur population et l'engouement pour les nouvelles cités ont eu un impact direct sur les quartiers historiques, qui ont connu des réalités plurielles (dégradation du centre historique, dévalorisation du patrimoine bâti, "muséification" des quartiers, etc.). Le bâti traditionnel dans les quartiers Zebda et Guitna a été affecté par le vieillissement et le délabrement, voire la disparition de certains éléments significatifs de sa richesse plastique et architecturale.

La ville de Tozeur connaît aujourd'hui une extension urbaine fulgurante ( $c f$. figure 1$)$, due essentiellement à l'importance accordée par les pouvoirs publics au tourisme saharien - qui s'est manifestée, d'une part, par la création d'une zone touristique $^{(3)}$ qui ne cesse de se développer et dont l'architecture hôtelière tente de s'inspirer du patrimoine traditionnel, surtout au niveau du traitement des façades, et, d'autre part, par la réhabilitation et la mise en valeur historique notamment du quartier Ouled El Hadef qui a conservé remarquablement, contrairement aux autres quartiers, sa trame urbaine intra-muros. L'architecture remarquable de ses ruelles et la proximité de l'oasis, qui garde toujours son système d'irrigation traditionnel ${ }^{(4)}$, se prêtent à une consommation touristique (Battesti, 2005). Les tentatives lancées par les autorités locales pour mettre en valeur les vieilles palmeraies en vue de répondre aux attentes des flux touristiques entrent dans la perspective d'insérer l'ensemble de la région dans un travail de conservation et de protection des sites historiques sahariens ${ }^{(5)}$. Cette nouvelle réalité urbaine commence à bouleverser, depuis quelques années, les pratiques et les rapports sociospatiaux des populations dans la ville (Puig, 2004). En effet, le patrimoine se découvre comme une ressource essentielle de régénération qui pourrait contribuer à l'amélioration de la qualité de vie urbaine. Aujourd'hui, Tozeur, que l'on considérait il n'y a pas longtemps comme un grand bourg rural, est une ville réinventée. Elle a enregistré un solde migratoire positif entre 1984 et 2008, contrairement à d'autres régions du Sud tunisien (cf. figure 2). 


\section{Figure | L Lisibilité des quartiers historiques} malgré le développement urbain

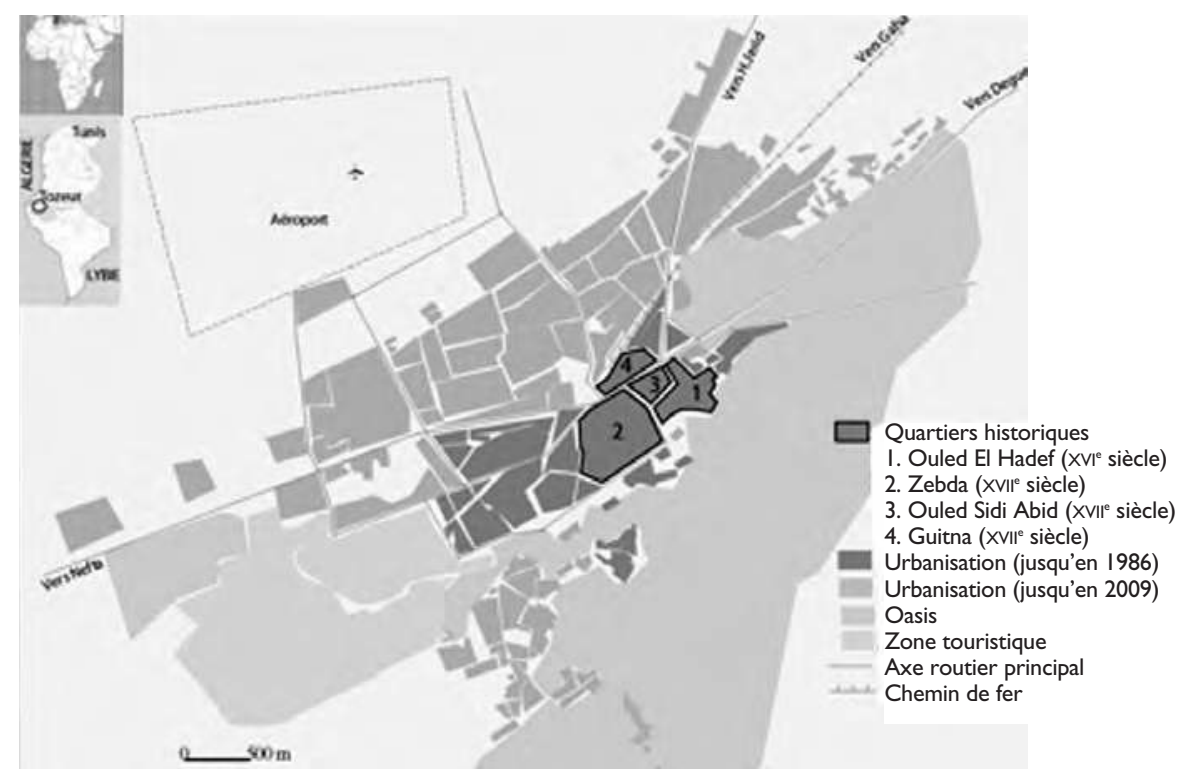

Cartographie : N. Dhaher, 2009.

\section{Figure 2 - Évolution de la population urbaine de Tozeur}

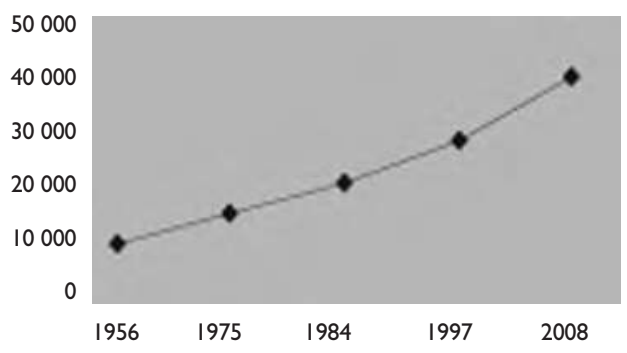

Source : Recensements de l'Institut national de la statistique, Tunis.

\section{Une architecture urbaine}

\section{bien particulière}

À l'échelle globale, l'identité générale de la ville de Tozeur est tout d'abord définie par sa situation entre l'oasis et l'espace désertique. Entre ces deux grandes entités paysagères, les quartiers historiques sont nés au bord de l'oasis. Empreinte d'une forte tradition culturelle, la partie centrale de la ville se confond avec ses quartiers anciens et notamment celui d'Ouled El Hadef, le plus important de la cité.

La logique de l'organisation spatiale des quartiers historiques, malgré une morphologie éclatée et une certaine fragmentation spatiale, obéit à une structuration particulière avec des caractéristiques urbaines, architecturales et paysagères qui dessinent en effet l'identité de la ville. D'ailleurs, en regardant le schéma du centre-ville, on constate qu'il est composé d'éléments aux aspects morphologiques plutôt différents : - les quartiers Ouled El Hadef et Guitna ont une structure irrégulière formée d'îlots qui sont une agrégation de mailles mais tenant compte du voisinage et de l'environnement. Les habitants de ces quartiers ont en fait les mêmes origines, ce qui a eu un effet sur la structuration du quartier ;

- le quartier Zebda, qui rassemble des familles d'origines différentes, présente une structure formée d'îlots juxtaposés caractérisés par leur alignement et leur orthogonalité relative. Cela est dû à un afflux massif de populations et au rythme accéléré de leur installation.

Le cadre de vie véhicule des valeurs culturelles et identitaires à travers un tissu composé de trois éléments principaux : la ruelle, la placette et l'impasse ( $c f$. figure 3). Ces éléments principaux de la structure des quartiers sont toujours en place malgré leur vétusté et n'ont pas changé, à l'exception de quelques modifications. Pour les entités qui ont conservé remarquablement leur trame urbaine intra-muros, tel que le quartier Ouled el Hadef, cette caractéristique a constitué un atout majeur pour le développement du projet de mise en valeur historique et de réhabilitation d'une partie notable du centre traditionnel - en 
vue de créer des produits touristiques susceptibles d'attirer une nouvelle clientèle et d'accroître la rentabilité économique du secteur.

La croissance récente de la ville de Tozeur, notamment dans les domaines agricole, administratif et touristique, a permis au centre de se développer, à travers des activités concurrentes et pas toujours compatibles avec la structure du centre ancien.

\section{Patrimonialisation \\ ET MISE EN TOURISME \\ DE L'ARCHITECTURE}

TRADITIONNELLE

La mise en valeur des quartiers historiques des villes du Sahara, notamment tunisien, s'est appuyée depuis des années sur l'exploitation du bâti traditionnel et des paysages du désert sans accorder une importance à la valorisation de l'héritage architectural. Cela a porté atteinte à l'urbanisation saharienne historique et au patrimoine. À la fin des années 1990, après avoir accordé la priorité au tourisme saharien, l'État provoque dans la ville de Tozeur un effort réflexif autour de la notion de patrimoine, destiné à systématiser l'offre touristique et à promouvoir un développement économique et culturel local.

\section{La sauvegarde du patrimoine : un enjeu culturel} et socio-économique

À la suite de cette prise de conscience des valeurs techniques, culturelles et sociales que recèle le

\section{Figure 3 - Une structure urbaine particulière}

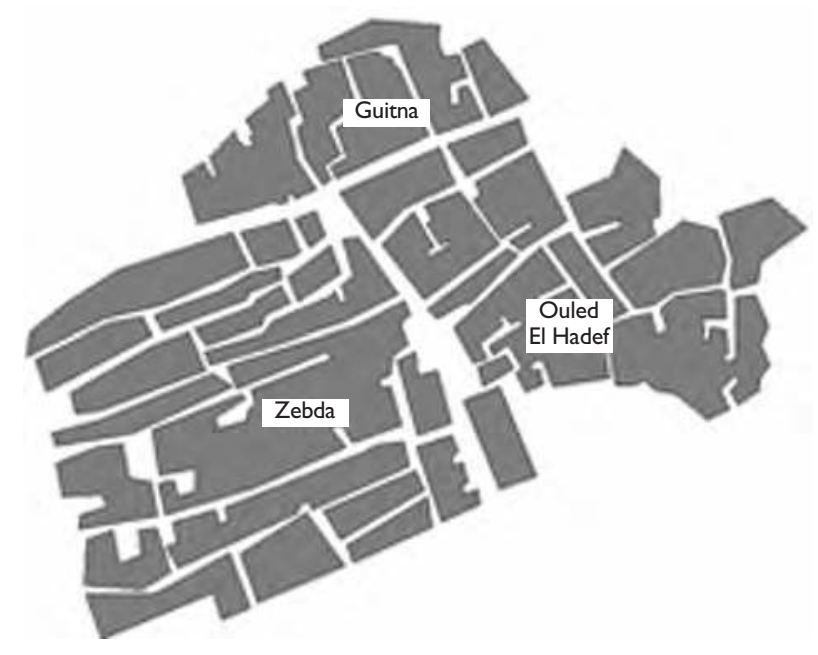

Cartographie : N. Dhaher, 2009. patrimoine, l'intégration de ce dernier dans le contexte urbain, économique et social contemporain est devenue un enjeu majeur pour l'aménagement et l'identité de la ville. Ainsi, les opérations de préservation et de réhabilitation se veulent une action salvatrice de poids non seulement pour la sauvegarde d'un patrimoine en péril, mais aussi pour son intégration dans le processus de développement socio-économique de la ville. Autrement dit, il faut qu'à la valeur d'usage, s'ajoute une valeur d'échange (Bonard et Felli, 2008). Certains pensent que, "pour qu'il y ait patrimonialisation, il ne suffit généralement pas que l'héritage ciblé ait acquis du sens pour un groupe, une collectivité et qu'il y ait une légitimation 'scientifique' par les spécialistes du patrimoine : il faut également que l'objet patrimonial puisse acquérir une valeur économique" (Veschambre, 2007, p. 2).
Plusieurs actions de mise en valeur ont été réalisées en fonction des différentes contraintes touchant l'état du bâti, l'occupation des parcelles, le type d'occupation, le caractère représentatif et la conservation de quelques éléments patrimoniaux.

Ainsi, le ministère du Tourisme s'est chargé de restaurer les façades et l'Institut national du patrimoine de restaurer les monuments. Pour sa part, la municipalité a mené depuis quelques années des opérations très limitées de réhabilitation de la voirie et des maisons et d'“embellissement" de la ville, épaulée en cela par une forte promotion de la réutilisation de la brique cuite et artisanale locale par la population. Toutefois, il convient de comprendre comment le caractère "marchandisé" du patrimoine tozeurois, lié à des politiques publiques de valorisation de l'héritage culturel et à sa mise en tourisme, s'incarne concrètement. 
Figure 4 - L'héritage architectural, une composante essentielle de la ville de Tozeur

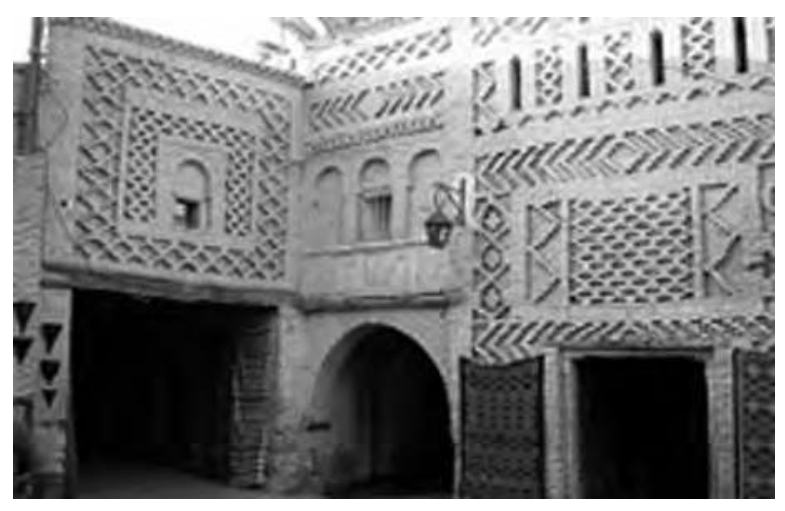

Dans les quartiers historiques de la ville, l'habitat traditionnel dégradé et voué à la disparition est devenu synonyme de pauvreté pour les populations qui y résident encore. Les occupants ne disposent pas de moyens financiers pour réaliser de nouvelles constructions. Avec l'inscription du centre historique de Tozeur par l'Unesco au "patrimoine mondial culturel" en 1988, une opération urbanistique et patrimoniale d'envergure a été déclenchée. Le centre urbain ancien de la ville a été soumis à des travaux de réhabilitation qui s'appuient sur la patrimonialisation. L'idée essentielle est de rendre le centre ancien plus attirant pour le tourisme en mettant en avant ses qualités historiques. Cette marchandisation n'est pas une stratégie ou une politique mais plutôt un constat et une conséquence.

Pour Gravari-Barbas (1998), les politiques patrimoniales urbaines contribuent à faire une ville idéologiquement correcte et esthétiquement attrayante en aplanissant les incohérences de l'histoire, l'enjeu étant en définitive d'en faire un produit quasi parfait, plus que la réalité elle-même.

Dans ce sillage, la brique cuite locale - qui est l'emblème de ce patrimoine - a fait l'objet d'un décret municipal: "Aucun édifice, aucune résidence, aucune construction ne se fera dorénavant sans le recours dans une proportion d'au moins $30 \%$ à la brique locale. ${ }^{(6)}$ " De là le tourisme commence à se présenter comme un atout pour la valorisation du patrimoine et permet son évolution à travers la fonction économique ( $c f$. figure 4).

Ce nouveau rôle dévolu au patrimoine a eu des effets bénéfiques sur l'environnement socio-économique : création de petits commerces, d'emplois permanents sur le site, etc.

Le centre de gravité de la ville, caractérisé par le quartier Ouled El Hadef et les axes alentour, est devenu tributaire des nouvelles articulations de ces espaces et de leurs temporalités. En effet, l'industrie touristique, de plus en plus dominante risque d'aggraver la fragilité de l'équilibre entre la vocation résidentielle et la multifonctionnalité des lieux. Les aménagements entrepris pour sauvegarder et mettre en tourisme le patrimoine dans la zone centrale semblent revaloriser cet espace, mais laissent présager des incohérences qui risquent de compromettre son existence. Plusieurs problèmes se sont ainsi posés à propos de cet espace, à savoir l'inadaptation du tissu traditionnel aux nouvelles fonctions du centre, la congestion du centre en matière de circulation, la rupture avec certaines formes urbaines et architecturales traditionnelles et la disparition de leur spécificité. Bref, “touristifier” s'est avéré une opération qui, à Tozeur, n'est pas exempte de difficultés et de risques. En effet, les actions de valorisation et de rénovation n'ont pas toujours profité à l'architecture traditionnelle, à ses matériaux et à ses savoir-faire. D'autre part, la structure morphologique du centre ancien de Tozeur s'est sensiblement modifiée. Elle s'est fortement densifiée en dehors de toute planification. Ce tissu ancien, souvent exclu des circuits modernes, voire "marginalisé", s'est trouvé réduit à des fonctions d'accueil et de logement d'une population majoritairement pauvre.

\section{Une mise en tourisme paradoxale}

Un grand débat anime actuellement le présent du patrimoine et le futur du tourisme. En effet, lorsque l'on met les notions de patrimoine et de tourisme en corrélation, celle- 
ci s'impose davantage comme un outil de développement économique territorial local. Il est vrai que la gestion du patrimoine bâti s'avère souvent délicate quand il s'agit de concilier la rentabilité économique avec la préservation des sites et des monuments historiques. Dans un pays comme la Tunisie, la valorisation touristique du patrimoine a toujours été confrontée à de nombreuses contraintes, relevant aussi bien du volume des investissements à consentir que du statut juridique des sites et monuments et surtout des logiques des acteurs locaux. Aujourd'hui, la ville de Tozeur est façonnée par différentes mobilités touristiques, commerciales et de loisirs. La mise en tourisme du patrimoine participe à sa revitalisation par le potentiel économique qu'il représente. Après sa restauration, le quartier bédouin d'Ouled El Hadef a connu une mixité de plus en plus prononcée en raison des nouvelles appropriations des lieux. Ce quartier, rebaptisé "ville traditionnelle", est révélateur de la transformation d'un lieu de vie en lieu de décor pour des besoins touristiques.

Avec le développement du secteur touristique, l'artisanat a connu un essor dans la ville. Cette réalité a provoqué une vague de transformation des houchs - maisons à patio ouvertes seulement vers l'intérieur - donnant sur l'axe principal en boutiques pour la vente des produits de l'artisanat local (cf. figure 5). Des maisons traditionnelles ont été transformées en restaurants ou en cafés pour répondre aux besoins des visi-
Figure 5 Ancien houch transformé en boutiques d'artisanat
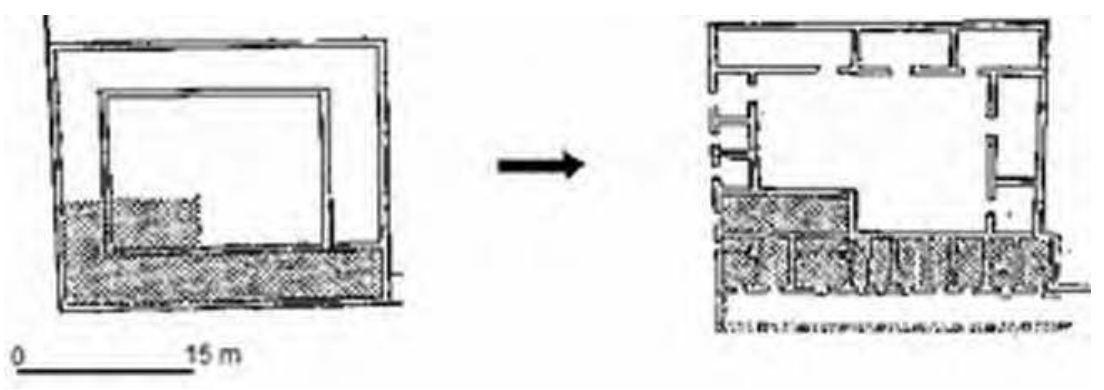

Figure 6 Ancien fondouk transformé en marché
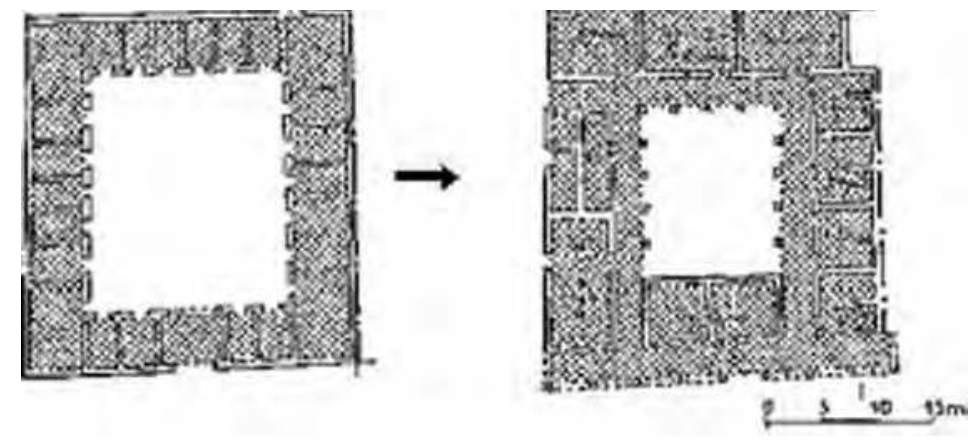

teurs et des touristes. Les patios ont été réduits pour obtenir le maximum d'espace sur l'axe principal, les galeries aménagées, etc. Les anciens foundouks ont été transformés en hôtels et en marchés avec introduction de nouveaux éléments (vitrines, baies vitrées, etc.) ( $c f$. figure 6).

Ces transformations ont été effectuées sans tenir compte de l'identité architecturale du tissu traditionnel (cf. figure 7). Elles ont affecté de façon importante l'aspect et la composition des façades. En général elles ont rompu la relation et l'équilibre originels entre les vides et les pleins, perturbé la verticalité, l'horizontalité et la hiérarchie dans l'ordonnancement des baies. À divers degrés, ces modifications ont aussi transformé le caractère de certaines typologies du patrimoine. Certaines d'entre elles, bien qu'apparemment légères, ont changé profondément l'aspect du bâti et engendré des pathologies variées, associées à des pratiques ignorant les particularités et les valeurs de l'architecture traditionnelle. Ces modifications, parfois très étendues à cause d'un manque de contrôle des agents administratifs et d'un cadre législatif inadéquat, s'imposent souvent par des voies réglementaires. Les causes, liées aussi à l'abandon de certains matériaux et techniques traditionnels, sont en réalité diverses ; l'adoption d'un code d'urbanisme marginalisant l'ar- 
Figure 7 Une transformation architecturale qui tourne le dos à l'identité des lieux
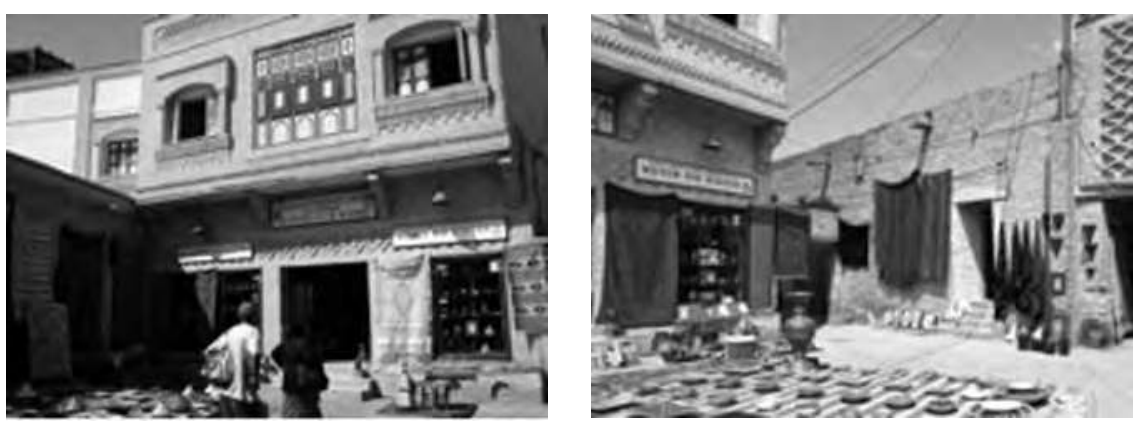

Figure 8 • L'avenue Bourguiba, principale rue commerçante dans la ville ancienne

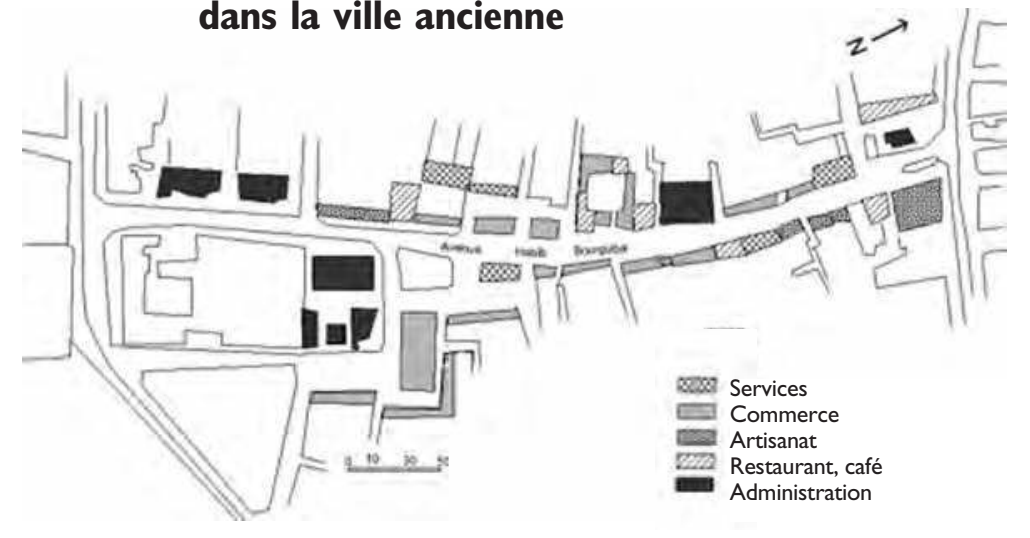

Cartographie : N. Dhaher, 2011.

Figure 9 - Certaines opérations d'extension et de restauration sont en déphasage avec la mémoire des lieux
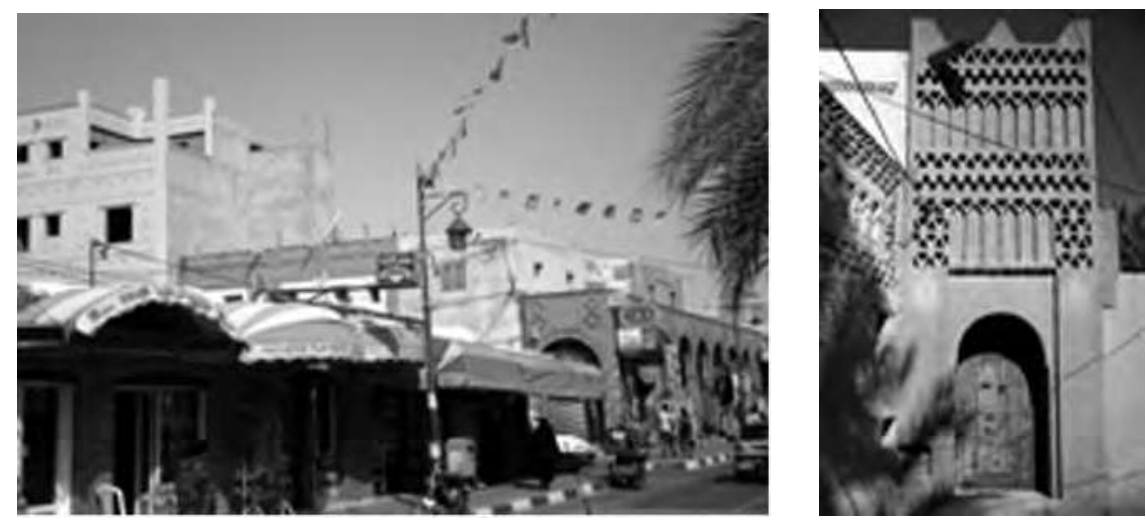

chitecture "introvertie" (maison à patio, sans façades extérieures) au profit d'une architecture "extravertie" en fait partie.

Le réaménagement des surfaces intérieures entre aussi parfois, du fait de la spéculation immobilière, dans les processus de densification des quartiers traditionnels. Cette réalité a entraîné un taux croissant d'urbanisation et de construction, un développement du parc automobile qui ont affecté aussi bien la ville que les quartiers historiques, tant dans leur organisation spatiale que dans leur morphologie. Des percées de plusieurs voies dans ces anciens quartiers, des "aérations", des élargissements de rues jusqu'au gabarit voiture ou le ceinturage étouffant des nouveaux quartiers sont autant de causes de défiguration de cette architecture.

Les différents changements du profil volumétrique du bâti traditionnel s'associent parfois à la disparition d'éléments essentiels de la typologie du patrimoine. C'est le cas, par exemple, du remplacement des sabbats (passages couverts) et des contrevents traditionnels, ou du pavage des ruelles. Très souvent, des problèmes structurels apparaissent, par augmentation des charges ou par déplacement des points porteurs, ce qui accélère la dégradation du bâti. Mais, également, les relations sociales traditionnelles, qui exerçaient un minutieux réglage des actes individuels, ont disparu et n'ont pas été remplacées dans les usages. Cet abandon culturel des pratiques alimente aussi ces trans- 
formations qui sont en train de se produire, la plupart du temps de façon irréversible (cf. figure 8).

Mais si les actions menées ont somme toute permis de respecter certains éléments de l'ancienne structure, comme l'homogénéité du parcellaire et certains traits originels, elles n'ont pas toujours réussi à s'accorder sur les types architecturaux qui peuvent s'intégrer harmonieusement au paysage urbain.

Ces types d'opérations de restauration semblent éloignés de ce que pourrait être une volonté de préserver le caractère authentique du patrimoine dans ces espaces. Le processus de conservation du patrimoine, qui ne parvient pas toujours à conserver la substance historique et l'aspect culturel et à sauvegarder les valeurs patrimoniales, n'a pas pu contrôler la manière dont ces espaces continuent d'évoluer. Alain Bourdin met en lumière le risque des politiques de patrimonialisation "d'aboutir à une mise en valeur généralisée, pauvre de signification et balisée par quelques éléments emblématiques caricaturaux" (Bourdin, 1996, p. 13). Pour Henri Lefebvre, "il est vrai que la ville persiste, mais dans un aspect muséifié et spectaculaire. L'urbain conçu et vécu comme pratique sociale est en voie de détérioration et peut-être de disparition" (2006, p. 56). D'ailleurs, l'un des problèmes de la mise en tourisme des quartiers historiques à Tozeur réside dans le fait que, le plus souvent, les espaces patrimoniaux ont été adaptés aux conditions d'habitabilité et de fonctionnement actuelles, ce qui a surtout entraîné des dysfonctionnements dans les pratiques de l'espace, les formes d'appréhension du temps et de l'espace par les touristes se superposant à celles des habitants permanents.

Certes, les espaces domestiques ont été soumis à de nombreuses évolutions qui témoignent de la diffusion de nouvelles valeurs urbaines. Mais certaines manœuvres des acteurs locaux - qui ne s'accordent pas avec l'identité locale tout en tentant de répondre aux représentations touristiques - conduisent parfois à une "folklorisation" du patrimoine soit par l'introduction de matériaux qui ne correspondent pas au statut de l'édifice, soit par la modification des structures de base qui corrige imparfaitement les défauts de l'architecture originelle.

La transformation dans les quartiers anciens a touché aussi bien le côté architectural que le côté spatial et urbanistique. C'est la dégradation du bâti par la nature fragile de la maçonnerie qui est alarmante. On peut voir des chantiers de réhabilitation utilisant des matériaux et des techniques étrangers à ceux de la région de Tozeur. Les textures des façades, qui s'apparentent à l'ornementation des tapis et des divers tissages locaux d'origine berbère et qui ont été modifiées en raison des effets du temps et de l'abandon, connaissent actuellement, sous l'effet du tourisme, des interventions bien souvent insensibles aux valeurs historiques du bâti ( $c f$. figure 9).

La mise en tourisme, qui semblait être le vecteur d'une prise de conscience de la valeur du patrimoine, prend de plus en plus la forme d'une "consommation culturelle" qui tend vers une banalisation et une perte progressive de l'identité patrimoniale locale. Hervé Barré $^{(7)}$ résume le dilemme en parlant d'un phénomène à deux faces, positive et négative : "Le tourisme est très paradoxal : c'est à la fois un prédateur et un sauveur du patrimoine. Le tourisme peut être à la fois la meilleure et la pire des choses pour le patrimoine." La situation actuelle à Tozeur n'est pas sans poser de problèmes. D'ailleurs, sous le prétexte de faciliter l'accueil d'un nombre croissant de touristes en vue de dynamiser l'économie locale, la réalisation d'opérations de réhabilitation et de rénovation et l'implantation d'équipements d'accueil mal adaptés et dénués de toute référence culturelle et architecturale visant tout simplement les bénéfices économiques - ont abouti à la banalisation de plusieurs édifices traditionnels.

\section{CONCLUSION}

La mise en tourisme du patrimoine, sa valorisation, sa gestion et sa protection représentent un enjeu culturel, social et économique pour le développement des territoires et contribuent à la lutte contre la pauvreté. Le tourisme ne se présente pas toujours comme une panacée. En effet, les responsables, qui affichent pourtant une ferme volonté de valoriser le patrimoine dans de nombreuses villes tunisiennes, ne 
disposent pas encore de programme d'action dans ce sens. La mise en tourisme du patrimoine à des fins de développement local doit être respectueuse de son identité et lui garder son authenticité : "Le tourisme est comme le feu, il peut cuire ta soupe ou brûler ta maison" (Furt et Michel, 2006). L'affirmation de l'authenticité doit s'opposer à la destruction et à la substitution par les œuvres grossières de remaniement (lentile, Bellanca et Naretto, 2008). La réalité tenace du rapport souvent inégal entre les touristes et les populations locales, et le déséquilibre entre ces dernières et les promoteurs touristiques invitent à penser de nouvelles formes de régulation. Car, dans cette course au touriste, les besoins culturels, récréatifs et même résidentiels de la population locale risquent de devenir secondaires. L'affirmation des villes comme centres d'activités, de développement et de tourisme dans un contexte fortement concurrentiel est liée à une bonne mise en valeur du patrimoine urbain (Bourdin, 1996). Il s'agit de coordonner la sauvegarde du patrimoine avec d'autres mesures, telles que la requalification de l'espace public en faveur de la population résidente, la gestion de la circulation, l'esthétique urbaine, etc. Cette coordination est à mener de façon judicieuse, c'est-à-dire en prenant en compte les dimensions temporelle et sociale des acteurs.

Les patrimoines architecturaux représentent pour les villes un potentiel de développement important. En effet, ils attirent les touristes et ils sont les témoins d'un savoirfaire local. Dans le contexte actuel de la concurrence touristique, le patrimoine culturel, qui prend de plus en plus un caractère marchand en tant que produit à valoriser économiquement, est devenu un enjeu majeur de l'attractivité touristique et du développement des territoires. Certes, la protection du patrimoine dépend pour beaucoup de son occupation, de sa fonctionnalité et de son rôle dans la société actuelle. Mais les acteurs publics et privés doivent comprendre les enjeux inhérents au fait de restaurer un quartier historique fréquenté par les touristes et de garantir sa survie, sa pérennisation et sa transmission à des générations futures. La volonté des acteurs locaux à Tozeur d'articuler le patrimoine au développement notamment économique de la ville nécessite une gestion qui permette de respecter les intérêts des communautés locales impliquées dans l'essor du tourisme et d'assurer une exploitation durable de ce patrimoine (Garat, Gravari-Barbas et Veschambre, 2008). L'enjeu est de savoir faire du tourisme le vecteur d'une prise de conscience de la valeur du patrimoine sans aboutir à sa banalisation rapide, contrairement à ce que l'on aperçoit aujourd'hui dans un tourisme incontrôlé qui s'accompagne d'effets pervers, tels que la "folklorisation" de ce patrimoine, pour répondre aux besoins des touristes (Babadzan, 200 I). Et cela met en cause l'une de nos responsabilités majeures qui réside dans l'obligation morale de léguer aux générations futures le patrimoine dont nous-mêmes avons hérité. Cette question est en effet encore émergente en Tunisie où l'articulation des actions locales et des politiques publiques reste problématique.

NOTES

( I) En 1529, El Hadef est venu de Biskra, en Algérie, habiter Tozeur et devenait le véritable chef de la ville. La famille d'El Hadef, d'origine arabe, s'est implantée dans un regroupement de structures qui consistait en des houchs accolés suivant une structure appuyée sur des rues étroites, des impasses et des placettes bahbouha.

(2) Le houch est la maison d'habitation traditionnelle ; son style et sa forme sont très particuliers. Les chambres et le séjour entourent une grande cour spacieuse, sorte de patio intérieur. Les chambres à coucher se composent d'une doukkana (endroit pour dormir en hiver), d'une salle d'eau et d'une ghorfa aux fenêtres donnant sur l'extérieur, qui est utilisée pour dormir dans la période d'été très chaude.

(3) Depuis le début des années 1990, le gouvernement donne la priorité au tourisme saharien. II finance la construction d'un aéroport international à Tozeur et de routes, et il autorise la construction d'une douzaine d'hôtels de grand standing pour attirer des touristes du monde entier vers des séjours clés en main.

(4) L'oasis de Tozeur est la seule à avoir maintenu son système d'irrigation traditionnel, échappant aux canalisations en béton (cf. Attia, 1983)

(5) L'atlas du gouvernorat de Tozeur 


\section{RECHERCHE • DOSSIER}

signale le travail de sensibilisation au sujet des espaces oasiens en tant qu'écosystème faisant partie du patrimoine culturel et écologique.

(6) Ces briques servaient jadis à construire entièrement les maisons, mais actuellement, devenant trop chères (60 millimes la brique) par rapport aux briques

industrielles, elles ne servent plus qu'à décorer les façades exposées sur la rue pour que Tozeur conserve son apparence historique. La production de la brique traditionnelle fait partie de l'économie locale. Le secteur emploie une vingtaine de familles.

\section{RÉFÉRENCES BIBLIOGRAPHIQUES}

Habib AtTIA, "Étatisation de l'eau dans les oasis du Jérid tunisien, lecture d'une dépossession", Annuaire de l'Afrique du Nord, vol. XXII, 1983, pp. 36I-375.

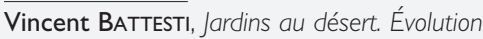
des pratiques et savoirs oasiens, Jérid tunisien, "À travers champs", Éditions IRD, 2005.

Alain BABADZAN, "Les usages sociaux du patrimoine", Revue électronique du Cerce, $n^{\circ} 2,2001$.

Yves BonARD et Romain FelLI, "Patrimoine et tourisme urbain. La valorisation de l'authenticité à Lyon et Pékin", Articulo - Journal of Urban Research, $n^{\circ}$ 4, 2008.

Alain BourdiN, "Sur quoi fonder les politiques du patrimoine urbain ?", Les Annales de la recherche urbaine, $n^{\circ} 72,1996$.

Jean-Marie FURT et Franck MICHEL,

Tourismes et identités, coll. "Tourismes et sociétés", L'Harmattan, 2006.

\section{Isabelle Garat, Maria Gravari-Barbas}

et Vincent Veschambre, "Préservation du patrimoine bâti et développement durable: une tautologie? Les cas de Nantes et Angers", Développement durable et territoires, mis en ligne le 3 mars 2008 [http://developpementdurable.revues.org/4 913].
(7) Hervé Barré est spécialiste du tourisme durable au Centre du patrimoine mondial de l'Unesco.
Maria Gravari-Barbas, "Villes et tourisme : images, espaces, acteurs", Norois, n 178 , 1998.

\section{Rosalba Ientile, Calogero BeLlancA et}

Monica NAREtTo, "Authenticité et sauvegarde de l'architecture vernaculaire, quel esprit du lieu"', contribution au $16^{\text {th }} /$ comos General Assembly and International Symposium, Québec, 2008.

Abdelfattah KASSAH, Les Oasis tunisiennes, aménagement hydro-agricole et développement en zone aride, "Série géographique", Ceres, 1996.

Henri LefeBVRe, "Métamorphoses planétaires", Manière de voir, n 89, 2006 (écrit en 1989).

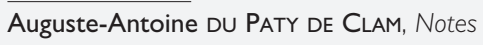
sur le Jérid tunisien, Archives diplomatiques, Rapports, études et conférences, bobine 5, 1890.

Nicolas PuIG, Bédouins sédentarisés et société citadine à Tozeur (Sud-Ouest

tunisien), Hommes et sociétés, 2004.

Vincent VesCHAMBRE, “Le patrimoine : un objet révélateur des évolutions de la géographie et de sa place dans les sciences sociales", Les Annales de géographie, n 656, 2007. 